\title{
Towards a First-Principles Evaluation of Transport Mechanisms in Molecular Wires
}

\author{
Susanne Kröncke and Carmen Herrmann* \\ Department of Chemistry, University of Hamburg, Martin-Luther-King-Platz 6, \\ 20146 Hamburg, Germany \\ E-mail: carmen.herrmann@chemie.uni-hamburg.de
}

\begin{abstract}
Understanding charge transport through molecular wires is important for nanoscale electronics and biochemistry. Our goal is to establish a simple first-principles protocol for predicting the charge transport mechanism in such wires, in particular the crossover from coherent tunneling for short wires to incoherent hopping for longer wires. This protocol is based on a combination of density-functional theory with a polarizable continuum model introduced by Kaupp et al. for mixed-valence molecules, which we had previously found to work well for length-dependent charge delocalization in such systems. We combine this protocol with a new charge delocalization measure tailored for molecular wires, and we show that it can predict the tunneling-to hopping transition length with a maximum error of one subunit in five sets of molecular wires studied experimentally in molecular junctions at room temperature. This suggests that the protocol is also well suited for estimating the extent of hopping sites as relevant, e.g., for the intermediate tunneling-hopping regime in DNA.
\end{abstract}

Date: June 27th 2020 


\section{Introduction}

The idea of using molecular wires as building blocks for nanoscale electronics has attracted the interest of both theoreticians and experimentalists, as it offers the possibility of establishing novel functionalities compared to conventional silicon-based electronics. ${ }^{1}$ For instance, the potential application of molecular wires as single-molecule insulators with even greater insulating properties than vacuum has been pointed out recently. ${ }^{24}$ Moreover, approaches for exploiting the spin-polarization properties of diamagnetic helical molecules, such as proteins or DNA, have been suggested in the past, where chiral-induced spin selectivity can be used to design more efficient water-splitting or memory devices. ${ }^{[-11}$ Besides potential technological applications, the field of molecular electronics is appealing due to its significance for fundamental science, offering insights into molecules under unusual circumstances. There are continuing efforts to study charge transport processes in biomolecules like peptides, enzymes and DNA, as they are of vital importance to every living organism, for example in the context of oxidative DNA damage. 12 14

A central question in such processes is the charge transfer mechanism. ${ }^{15}[19$ From conductance experiments on molecular wires built of a varying number of repeating monomer units, two main transport regimes have been identified, coherent tunneling and incoherent hopping. $115 \mid 16$ One of the main factors determining the predominant charge transport mechanism is the molecular length. A crossover from the tunneling to the hopping regime is frequently identified as a sudden reduction of length dependence of conductance for wires longer than around 3 to $4 \mathrm{~nm} \cdot \frac{15 / 16}{10}$

In the tunneling regime, the charge is transported coherently in a one-step process, while essentially not spending any time on the molecular bridge. $\frac{120}{120}$ This transport mechanism is characterized by an exponential dependence of the conductance on molecular length. In contrast, as the molecule becomes longer, the transition to incoherent hopping is marked by a linear dependence of the conductance on the length in accordance with Ohmic behavior.115/16 
As the latter process is strongly dependent on temperature in contrast to tunneling 1 , the transition is also marked by a change in temperature dependence. ${ }^{1}$ In the hopping regime, the charge transport timescale approaches the one of molecular vibrations, where dephasing processes associated with electron-phonon coupling can result in the formation of polarons, i.e. charge-localized deformations on specific molecular subregions. $\frac{120122}{12}$ As a consequence, the charge migrates through the molecule by subsequently moving through so-called hopping sites. Experimental data also point to an intermediate regime, in which hopping sites extend over large portions of the system, e.g. in $\mathrm{DNA}^{23} \sqrt[26]{26}$

The groups of Elstner and Kleinekathöfer presented a comprehensive multiscale approach to the description of charge transport in molecular junctions without the need of assuming an underlying transport mechanism a priori.2728 Related approaches have been put forward in the context of charge transport in organic crystals. $\frac{29}{35}$ There is also a substantial body of work on such comprehensive approaches in molecular junctions employing simple parametrized model Hamiltonians. ${ }^{25136}$ 43 However, charge delocalization in molecules can depend strongly on choices for approximations in the electronic structure description. 44 ? -48 If an accurate first-principles treatment of the electronic structure is desired, such comprehensive schemes would be computationally quite expensive. It also requires a computational implementation combining electronic structure calculations, $\frac{49150}{}$ Greens function techniques 51152 and molecular dynamics simulations. ${ }^{53154}$ For a computationally efficient and simple-to-implement first-principles description of charge transport processes, the identification of the crossover length can therefore be a valuable alternative, as it allows assuming either of the two transport regimes, leading to simpler theoretical descriptions of transport.

Here, we are aiming at a predictive approach for crossover lengths that is computationally efficient and easily applicable to any kind of molecular wires. The methodology presented in this study relies on a connection between the predominating charge transport mechanism and

\footnotetext{
${ }^{1}$ Temperature-dependence in tunneling through molecular junctions has been measured, but has been attributed to temperature-induced structural changes of the electrodes 21
} 
charge localization properties: One could link tunneling transport to a completely delocalized charge, while increasing charge localization could be associated with hopping transport.20122 The charge is then predominantly located on specific subregions of the molecule, where polarons are formed. $\frac{15155156}{}$ The molecular wires under study are considered in their singly oxidized radical-cationic state, as holes rather than electrons dominate the transport in the wires under study here. In contrast, there are much fewer examples of charge transport through molecular wires dominated by electrons (corresponding to radical-anionic organic mixed-valence species ${ }^{57}$ or electron-deficient thiophene-1,1-dioxide oligomers) .58

In a previous study on first-principles approaches, we validated different computational protocols based on Kohn-Sham density-functional theory (KS-DFT) regarding their capability of describing length-dependent charge localization in comparison with experiments for organic mixed-valence systems. $\frac{48}{4}$ This type of donor-bridge-acceptor systems has the advantage of representing atomistically better-defined model systems than molecular junctions, while still being closely related to them as the electron transfer properties as expressed in their Robin-Day class are also length-dependent. ${ }^{5960}$ We found that, in contrast to other DFT protocols, a combination of the B1LYP hybrid functional with $35 \%$ Hartree-Fock exchange (BLYP35) and a polarizable continuum model (PCM), previously proposed by the group of Kaupp, ${ }^{44} \sqrt[46]{ }$ works well in this case. ${ }^{\sqrt[48]{8}}$ This was even though it can be assumed that entropic effects are playing an increasingly important role for the degree of charge localization in molecular wires as they get more flexible with increasing length, thus leading to a greater variety of possible structures with potentially different charge localization properties. In this study, we want to check the transferability of the validated DFT protocol to predicting transport mechanisms in molecular junctions with the primary goal of establishing an easily applicable and efficient, yet predictive approach based on first-principles.

If our approach succeeds, it will most likely be due to error compensation, as it has proven very effective in vibrational spectroscopy, where a systematic good agreement between 
experimental and harmonic wavenumbers calculated with the BP86 exchange-correlation functional was found due to the partial compensation of the harmonic approximation by

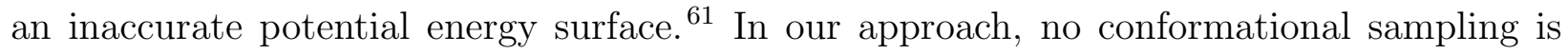
performed, and therefore molecular fluctuations are neglected.

Furthermore, it lacks an explicit description of electronic effects arising from the electrodemolecule-interface, which are particularly important in self-assembled monolayers (SAMs) due to the formation of dipole layers. ${ }^{62}$ 64 Since molecular conductance experiments are often performed on SAMs where molecular wires are closely packed, it is likely that the degree of charge localization is affected by the local environment, for example by charge-stabilizing effects arising from adjacent wires. In our simulations, environmental effects from a solvent or from adjacent molecular wires are approximated via a PCM.

Here, we intend to compensate these effects by the choice of the approximate exchangecorrelation functional and electrostatic embedding via PCM in order to correctly predict transport mechanisms, even though only single molecular structures at $0 \mathrm{~K}$ are considered $:^{2}$ We aim at the prediction of the tunneling to hopping crossover observed in molecularconductance experiments on conjugated organic wires $\frac{15}{19}$ on the basis of our static DFT approach. $\stackrel{46 / 48}{ }$ In Section 2, we describe our procedure of assessing and quantifying the degree of charge delocalization in more detail. In Section 3, we present our results from DFT calculations on charge localization properties for a variety of molecular wires and compare them with respect to their agreement with transition lengths from experiments. A conclusive summary of our results is provided in Section 4, where our findings are discussed in the light of possible applications and limitations of the method.

\footnotetext{
${ }^{2}$ However, as was pointed out by Elstner and Kleinekathöfer, caution needs to be exercised when choosing the effects that are to be included in the calculations, since the partial compensation or neglect of particular components might alter the description of the system markedly. For example, in a study on hole transport through DNA, it was shown that the polarization of the environment via the electric field and the relaxation of the electronic structure compensate each other to a certain extent as they induce opposite effects on the site energies of nucleobases. It therefore has to be kept in mind that there is a complex balance and counterplay of individual effects entering the theoretical description of charge transport processes. 2728
} 


\section{Assessment of Charge Delocalization}

\subsection{Definition of Charge Delocalization}

To investigate hole transport in the radical cationic molecular wires under study, we evaluated different approaches of determining the degree of charge delocalization in a well-defined manner. As the free electron and the excess positive charge are located on the same position of the radical cationic systems studied here, and free local spins are less basis-set dependent than local charges, $\frac{65}{6}$ the assessment of localization is based on the analysis of the local spin density. In our previous studies on organic mixed-valence systems, the ratio between the local charges on the donor and acceptor moieties connected by a bridge served as a measure for the degree of charge localization, where complete charge delocalization would be expressed by a ratio of one, whereas a ratio of zero would indicate a fully localized charge on one redox centre. ${ }^{48}$ Since here, the redox centers are replaced by non-redox-active anchoring groups, we define the degree of charge delocalization $r_{\text {deloc }}$ by relating the smallest possible subregion on which a suitably chosen large percentage of spin density is located to that percentage,

$r_{\text {deloc }}=\frac{\text { Fraction of smallest possible subregion on which } x \% \text { of spin density are located }}{x / 100}$,

where the percentage of spin density $x \%$ is chosen such that it represents a majority, e.g. $70 \%$, but not $100 \%$ as this would in practice almost always need the full molecule to be included into the subregion. In case of complete delocalization, the spin would be evenly distributed across the entire molecule, while with growing localization, the majority of the spin density would be located on an increasingly smaller fragment of the molecule. Therefore, a molecule would be described as fully delocalized in case of a ratio of one, where for example $70 \%$ of the spin density are spread across $70 \%$ of the molecule, and so on, leading to $r_{\text {deloc }}=1$. In contrast, increasing localization results in a decrease of the ratio 
due to the subregion hosting the predefined percentage of the spin density becoming smaller. Ideally, a semi-localized molecular wire right on the borderline between localization and delocalization, where (in an idealized case) 100\% of the spin density populates around one half of the molecule, would be characterized by a ratio of $r_{\text {deloc }}=0.5$. One could therefore set this value as a cut-off for defining a system as predominantly localized or delocalized, depending on $r_{\text {deloc }}$ being smaller or larger than 0.5 , respectively.

Since we will see in the following that one can hardly define a clear cut-off value for the prediction of the crossover from delocalization to localization that is consistently valid across a broad range of molecules, we rather assigned a molecule to either of the regimes based on the similarities of $r_{\text {deloc }}$ for consecutive wires. Accordingly, a relatively strong decrease of $r_{\text {deloc }}$ from a shorter to a longer wire would indicate distinctly stronger localization in the latter species, particularly if the values for $r_{\text {deloc }}$ at smaller and larger molecular lengths than these two wires would be relatively stable. In principle, one could set any value between 50 and 100 percent as the majority of the spin density, provided that it is not too close to any boundary of this range. We therefore tested several threshold values to evaluate the effect on the calculated delocalization measure and compared the predicted crossover lengths from different thresholds with the experiments.

\subsection{Illustrating the Charge Delocalization Measure on the Exam- ple of $\mathrm{OPTI}_{n}$ Wires}

In Figure 1, the procedure of assessing the degree of delocalization is illustrated on the example of the shortest member $\left(\mathrm{OPTI}_{4}\right)$ of a series of conjugated oligophenylene-thiopheneimine $\left(\mathrm{OPTI}_{n}\right)^{3}$ wires, investigated by Frisbie and coworkers. $\frac{15}{15}$ The local spin density as obtained from natural population analysis (NPA) $)^{66}$ is plotted per atom along the junction,

\footnotetext{
${ }^{3}$ Here, $n$ denotes the number of monomer units present in the molecular wires according to Figures 3 and 6 , including the anchoring group(s).
} 
where the subregions hosting the majority of the spin density are highlighted in different shades of yellow according to the indicated threshold.

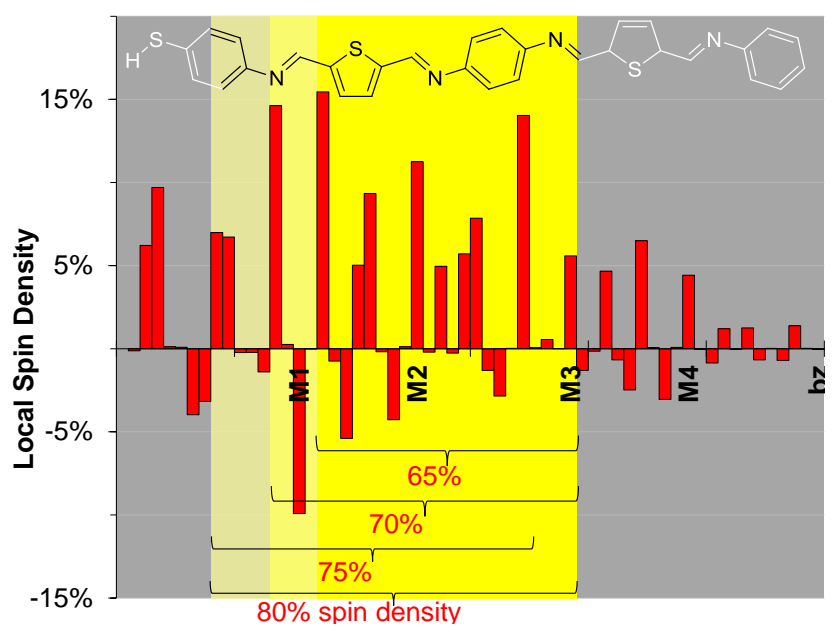

Figure 1: Local spin density distribution from NPA per atom along the molecular backbone of $\mathrm{OPTI}_{4}$ (sum over all local spin densities =1), optimized with the BLYP35 functional and Ahlrich's def2-TZVP basis set in thiophene (PCM). The smallest possible subregions containing a predefined fraction of the local spin density (65-80\%) used for the calculation of the delocalization measure $r_{\text {deloc }}$ according to Equation (1) are highlighted in different shades of yellow. Monomer units including the anchoring group according to Figure 3 are indicated by M1-M4 (bz=benzaldimine terminus).

In Figure 2, the calculated delocalization measure $r_{\text {deloc }}$ obtained from Equation (1) for $\mathrm{OPTI}_{n}$ wires is plotted as a function of the number of subunits for different fractions of the spin density, where the experimental crossover between tunneling and hopping from $\mathrm{OPTI}_{6}$ to $\mathrm{OPTI}_{7}$ is indicated by the black dotted line.

We predicted the crossover from the change in the delocalization measure $r_{\text {deloc }}$ between subsequent wires, marked by red bars in Figure 2. Therefore, molecular wires were considered in the same transport regime in case $r_{\text {deloc }}$ decreased continuosly by the same amount or less, while the crossover was determined from the greatest change of $r_{\text {deloc }}$ relative to subsequent wires. The crossover predicted from theory is indicated by a second dotted line, coloured according to the agreement with experiments, see Figure 2, As mentioned earlier, the crossover was expected to occur approximately around $r_{\text {deloc }}=0.5$, which was used as secondary criteria in case the evaluation based on the change of $r_{\text {deloc }}$ was not definite. 

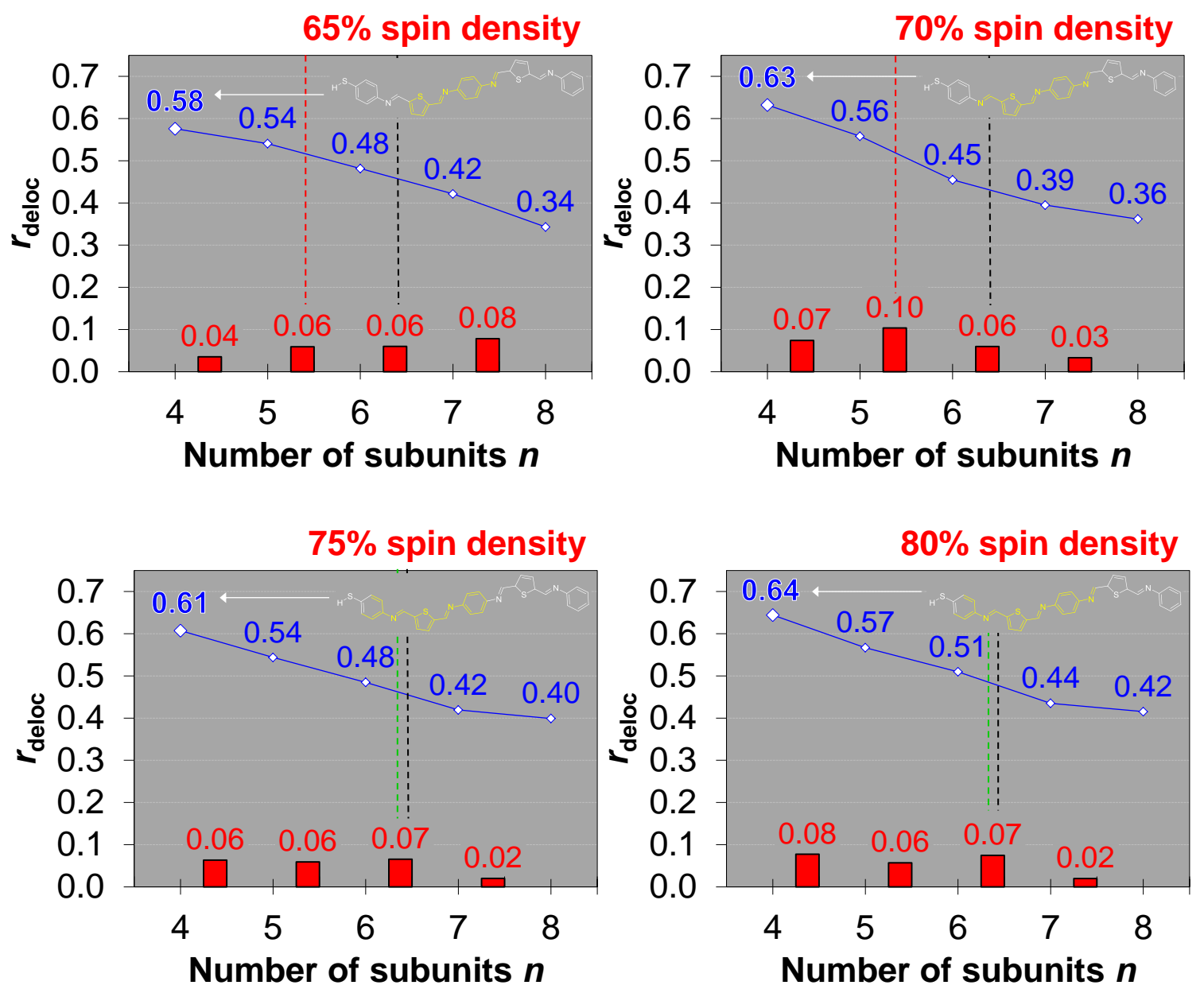

Figure 2: Delocalization measure $r_{\text {deloc }}$ of $\mathrm{OPTI}_{n}$ wires calculated according to Equation (1) as a function of the number of subunits $n$, see Figure 3. Molecular structures were optimized with the BLYP35 functional and Ahlrich's def2-TZVP basis set in thiophene (PCM). The selection of each subregion based on different thresholds for the spin density is illustrated by the Lewis structure of $\mathrm{OPTI}_{4}$. The delocalization measure is indicated by the blue line, the corresponding change in $r_{\text {deloc }}$ from subunit $n$ to $n+1$ is indicated by red bars. The experimental crossover from tunneling to hopping is marked by the black line. Coloured lines indicate the theoretically predicted crossover and its agreement with experiments (green: good; red: poor)

As can be seen from the plotted delocalization measure based on a threshold of $65 \%$ and $70 \%$ spin density, the crossover is predicted between $\mathrm{OPTI}_{5}$ and $\mathrm{OPTI}_{6}$ in each case and therefore one monomer unit earlier than from experiments. While in the former case, $r_{\text {deloc }}$ decreases relatively constantly within a range of 0.04 to 0.08 , a more distinct change in $r_{\text {deloc }}$ by 0.1 at most is observed in case of a higher threshold of $70 \%$. 
In contrast, the crossover predicted from a $75 \%$ and $80 \%$ threshold for the spin density is in good agreement with experiments and the changes in $r_{\text {deloc }}$ are qualitatively similar in both cases. Here, a relatively strong change in $r_{\text {deloc }}$ of 0.07 marks the crossover between $\mathrm{OPTI}_{6}$ and $\mathrm{OPTI}_{7}$, while $r_{\text {deloc }}$ only changes by 0.02 at longer lengths between $\mathrm{OPTI}_{7}$ and $\mathrm{OPTI}_{8}$, suggesting them being in the same transport regime.

While for the $\mathrm{OPTI}_{n}$ wires better agreement with the experiments was obtained in case of higher thresholds for the spin density, we could not deduce a clear trend of this being generally true for any kind of wires. In three out of five cases, the same results were obtained for all of the tested thresholds, while differences regarding the predicted crossover were observed only in case of $\mathrm{OPTI}_{n}$ and $\mathrm{OAE}_{n}$ wires (see Supporting Information, Section S3.2). For example, less agreement with the experiments was obtained for $\mathrm{OAE}_{n}$ wires when a threshold of $75 \%$ spin density was applied. However, best agreement was obtained consistently across all five tested series when a threshold of $80 \%$ spin density was applied, which therefore provides the basis for our analysis of the following results. A comparative table of the performance of different methods in predicting the tunneling-hopping crossover from experiments is provided in the Supporting Information (Section S3.1, Table S3), where also a slightly different approach in determining the subregion is presented, where the wires are fragmented into uniformly sized monomer units that are successively included into the subregion until the minimum percentage of spin density is reached (see Section S3.4 and S3.5 for results). In addition to the assessment of charge delocalization based on the calculated delocalization measure, we visualized the corresponding spin density distributions of the molecular wires under study. Ideally, the quantitative measure defined by Equation (1) should reflect our intuitive classification based on a visual assessment of these spin densities, which will be checked in the following. 


\section{Predicting Length-dependent Crossover from Static DFT Calculations}

To validate the capability of the BLYP35+PCM protocol in correctly describing charge transport mechanisms, we applied it to the calculation of charge localization properties of conjugated wires that previously had been investigated in molecular conductance experiments by various groups. $\frac{15-19}{19}$

First, our computational approach to predicting the length-dependent crossover from DFT is thoroughly discussed on the example of the thiophene-based $\mathrm{OPTI}_{n}$ wires, investigated by Frisbie and coworkers. $\frac{15}{15}$ these cases, our protocol turns out to work perfectly. We briefly compare our findings to related molecular structures, the $\mathrm{OPI}_{n}$ and $\mathrm{OAE}_{n}$ wires,

investigated by Frisbie and by Wandlowski and coworkers, respectively, $\stackrel{16119}{10}$ for which the protocol also works well. Second, we present our computational results on molecular wires where the degree of charge delocalization predicted from theory deviates to some extent from the experiments, the $\mathrm{ONI}_{n}$ and $\mathrm{OPE}_{n}$ wires, investigated again by Frisbie and coworkers and by the group of Wang, respectively. $\frac{17118}{1}$ Finally, we discuss the results obtained from our DFT calculations in the light of capabilities and possible limitations of our approach in a conclusive summary.

\section{1 $\mathrm{OPTI}_{n}$ Wires and Other Successful Cases - A Closer Look}

\subsection{1 $\mathrm{OPTI}_{n}$ Wires}

In conductive-probe atomic force microscopy (CP-AFM) experiments by Frisbie and coworkers at room temperature, a length-dependent transition from tunneling to hopping was observed in junctions based on $\mathrm{SAMs}_{\mathrm{s}}$ of $\mathrm{OPTI}_{n}$ wires up to $6 \mathrm{~nm}$ in length (see Figure 3), at a molecular length of approximately $4-5 \mathrm{~nm}\left(\mathrm{OPTI}_{6}\right.$ to $\left.\mathrm{OPTI}_{7}\right) \frac{[15}{,}$ see Table 1 . 
Our DFT calculations are consistent with the experimental crossover length: Distinctly higher localization is observed for the longer wires $\mathrm{OPTI}_{7}$ and $\mathrm{OPTI}_{8}$ when compared to the shorter wires. In Figure 3 , the local spin density per atom computed with the BLYP35 functional is depicted for each molecular wire next to the corresponding subregions of highest spin density, as defined in Section 2, marked in yellow. Since the experiments were carried out on SAMs in vacuum, the PCM for thiophene was employed during optimizations to model environmental effects arising from adjacent wires on charge localization.

As can be seen from Figure 3, the spin density is rather delocalized for the first three members of the series, therefore suggesting $\mathrm{OPTI}_{4}$ to $\mathrm{OPTI}_{6}$ belonging to the tunneling regime. This assumption is confirmed by the calculated delocalization measure according to Equation (1) ranging from 0.64 to 0.51 from $\mathrm{OPTI}_{4}$ to $\mathrm{OPTI}_{6}$. More importantly, a sudden drop of $r_{\text {deloc }}$ down to 0.44 indicates a distinctly increasing degree of charge localization for $\mathrm{OPTI}_{7}$, where most of the spin density is localized on a subunit comprising roughly three monomer units. The same delocalization length is observed for the longer $\mathrm{OPTI}_{8}$, characterized by an even lower $r_{\text {deloc }}$ of 0.42 , although differently located three-ring registers are suggested to be involved in the transport for the two species.

The fact that the length-dependent transition from tunneling to hopping can be predicted based on charge localization properties from static DFT calculations, where environmental effects are entering the model but conformational sampling and dynamics are lacking, is quite remarkable since the number of structures with different spin localization patterns likely rises with growing molecular length due to an increasing number of possible conformations. Hence, although other transport pathways may exist involving not only three- but also one- and tworing registers, as pointed out by Frisbie and coworkers,, 15 the static picture considered in this approach is sufficient for reasonably describing the crossover length for these molecular wires and therefore has predictive character.

While the inclusion of environmental modelling is found to be highly important for 

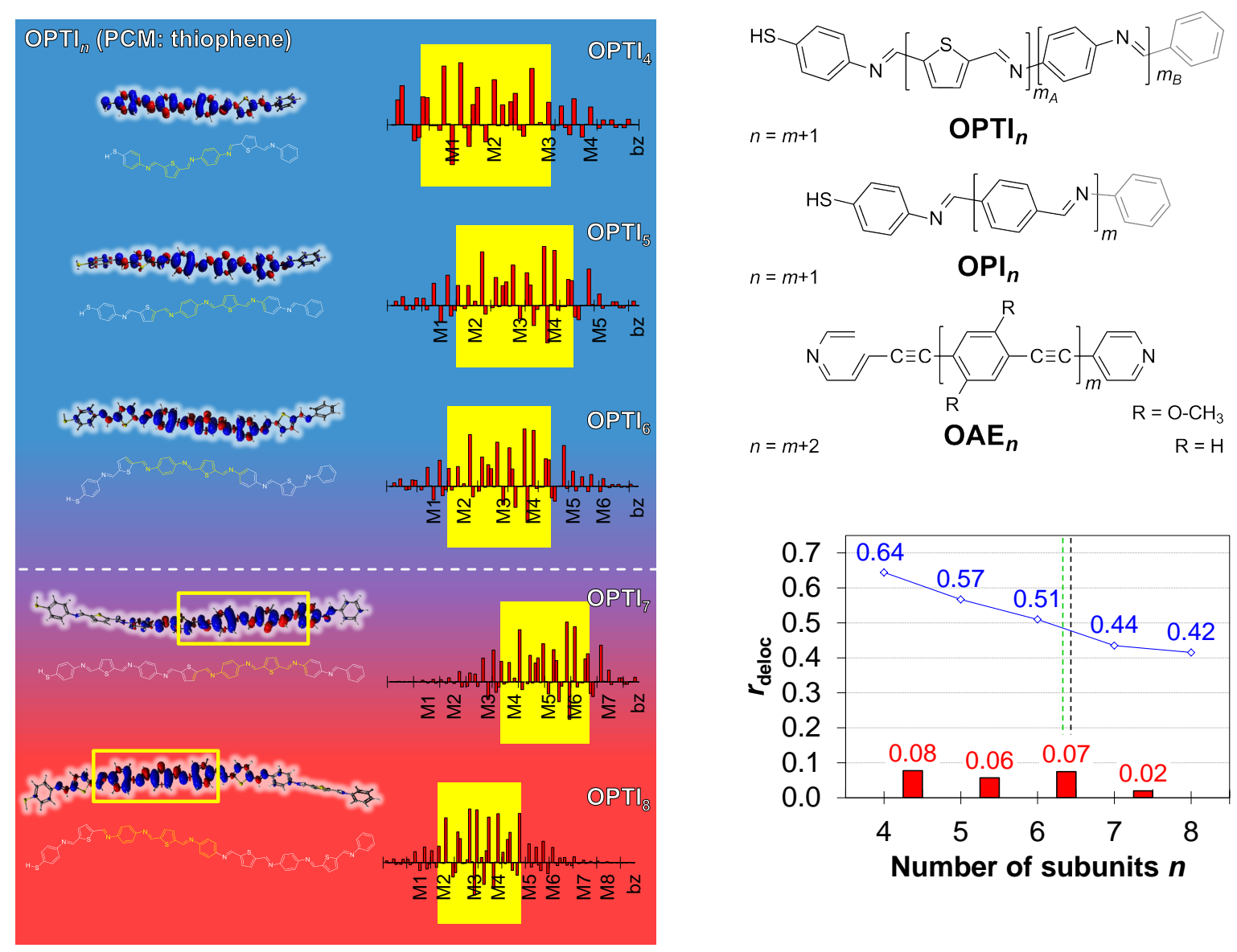

Figure 3: Left: Radical cationic $\mathrm{OPTI}_{n}$ wires optimized with the BLYP35 functional and Ahlrich's def2-TZVP basis set in thiophene (PCM). Spin densities and chemical structures of the molecular wires are shown with the local spin density per atom along the molecular backbone (total amount of local spin density $=1$ ). Subregions hosting the majority of the spin density (80-85\%) are highlighted in yellow. The degree of charge delocalization is coded by the background colour (charge delocalization: blue; increasing localization: red). The experimental crossover is indicated by the white dotted line. Top Right: Chemical structures of molecular wires, for which our protocol works well. Bottom Right: Calculated delocalization measure $r_{\text {deloc }}$ as defined by Equation (1) for molecular wires as a function of the number of subunits $n$ (blue line) and the corresponding change in $r_{\text {deloc }}$ from subunit $n$ to $n+1$ (red bars). Black dotted line: Experimental crossover from tunneling to hopping. Green dotted line: Theoretically predicted crossover based on the calculated delocalization measure (percentage of spin density: 80-85\%).

the correct description of charge localization properties, the simulation of the electrode by attaching one or three gold atoms to the molecular termini impairs the predictive potential of the method. Simulating the environment and the gold substrate at the same time leads to extensive overlocalization, as shown on the example of $\mathrm{OPTI}_{n}$ wires (see Supporting In- 
formation, Figures S17, S19 and Discussion in Section S4.4). Consequently, a more realistic description closer to the experiment, such as the electrode contact and environment, does not necessarily provide a better prediction of the crossover length, as previously pointed out by the groups of Elstner and Kleinekathöfer. ${ }^{2728}$ In particular the accurate first-principles description of molecule-metal interfaces in general is not trivial due to the formation of dipole layers in this region, which is particularly important in case of SAMs. 62 6467-74

\subsection{2 $\mathrm{OPI}_{n}$ and $\mathrm{OAE}_{n}$ Wires}

Since the BLYP35+PCM protocol worked well to predict the crossover length in case of the $\mathrm{OPTI}_{n}$ wires, we applied it to structurally related conjugated wires. A length-dependent crossover from tunneling to hopping was equally identified on SAMs of oligophenyleneimine $\left(\mathrm{OPI}_{n}\right)^{19}$ wires up to $7 \mathrm{~nm}$ long at a molecular length of approximately $4 \mathrm{~nm}\left(\mathrm{OPI}_{5}\right.$ to $\mathrm{OPI}_{6}$ ) by the group of Frisbie. $\frac{19}{19}$ Similarly, a crossover to hopping was observed for oligoaryleneethynylene $\left(\mathrm{OAE}_{n}\right)$ wires longer than $3 \mathrm{~nm}\left(\mathrm{OAE}_{5}\right.$ to $\left.\mathrm{OAE}_{6}\right)$ by Wandlowski and coworkers, where experiments were performed on single molecules in solution up to $6 \mathrm{~nm}$ long and functionalized on both termini with pyridyl-groups for the attachment to gold leads ${ }^{16}$ (see Figure 3 for chemical structures and Table 1 for comparisons of crossover lengths). The spin density distributions of $\mathrm{OPI}_{n}$ and $\mathrm{OAE}_{n}$ wires are provided in the Supporting Information (Section S1, Figure S1).

In Figure 4, the calculated delocalization measure as defined by Equation (1) is plotted for $\mathrm{OPI}_{n}$ and $\mathrm{OAE}_{n}$ wires as a function of molecular length. Since the experiments on $\mathrm{OPI}_{n}$ wires were performed on SAMs in vacuum, the PCM for benzene was employed during optimizations to model effects of adjacent wires on charge localization, while the PCM for tetrahydrofuran was employed to model the solvent in case of $\mathrm{OAE}_{n}$ wires.

For $\mathrm{OPI}_{n}$ and $\mathrm{OAE}_{n}$ wires, our DFT calculations are in good agreement with the ex-

perimental crossover from tunneling to hopping. When comparing $\mathrm{OPI}_{n}$ wires of different 

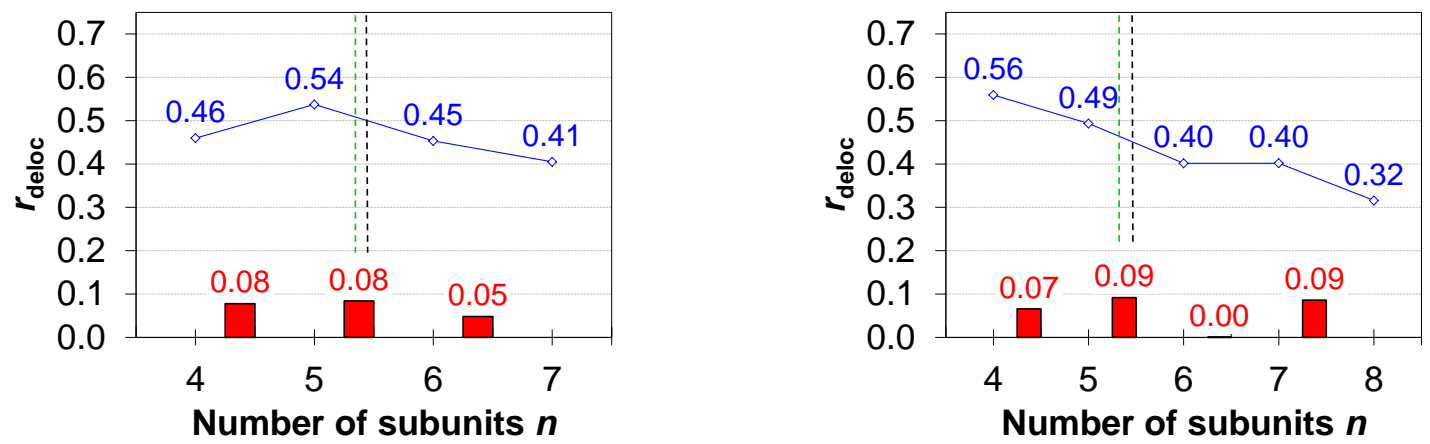

Figure 4: Calculated delocalization measure $r_{\text {deloc }}$ as defined by Equation (1) for OPI (left) and OAE (right) radical cationic wires (see Figure 3) as a function of the number of subunits $n$ (blue line) and the corresponding change in $r_{\text {deloc }}$ from subunit $n$ to $n+1$ (red bars). Black dotted line: Experimental crossover from tunneling to hopping. Green dotted line: Theoretically predicted crossover based on the calculated delocalization measure (percentage of spin density: $80-85 \%$ ).

lengths, a relatively strong decrease of $r_{\text {deloc }}$ between $\mathrm{OPI}_{5}$ and $\mathrm{OPI}_{6}$ from 0.54 to 0.45 clearly indicates increasing charge localization. Interestingly, slightly stronger localization is predicted for the shorter $\mathrm{OPI}_{4}$ when compared to $\mathrm{OPI}_{5}$. For the longer $\mathrm{OPI}_{7}, r_{\text {deloc }}$ decreases much more slightly down to 0.41 , suggesting the latter two species being in the same transport regime.

For the $\mathrm{OAE}_{n}$ wires, a distinctly higher degree of charge delocalization is observed for the shorter species $\mathrm{OAE}_{4}$ and $\mathrm{OAE}_{5}$ when compared to longer ones. Here, increasing charge localization is indicated between $\mathrm{OAE}_{5}$ and $\mathrm{OAE}_{6}$ by the highest change in $r_{\text {deloc }}$ from 0.49 to 0.40 , being the same for the longer $\mathrm{OAE}_{7}$.

Concluding these results, the BLYP35+PCM protocol is capable of predicting the lengthdependent crossover from tunneling to hopping as observed in conductance experiments on the molecules under study. Moreover, in any case of these wires the crossover is predicted to occur around a value of 0.5 for the delocalization measure. Consequently, the degree of charge localization as deduced from the calculated delocalization measure serves as a valuable tool for evaluating the underlying transport mechanisms in these molecular wires. 


\section{$3.2 \mathrm{OPE}_{n}$ and $\mathrm{ONI}_{n}$ Wires - Borderline Cases}

To put our DFT approach on more solid ground, we applied it to molecular wires similar in structure to the former species, the oligonaphthalene-fluorene-imine $\left(\mathrm{ONI}_{n}\right)$ and oligoparaphenylene-ethynylene $\left(\mathrm{OPE}_{n}\right)$ wires (see Figures 5 and 6 , investigated by the groups of Frisbie and Wang.17+19 Interestingly, these molecular wires provide an example of situations where the calculated localization properties point less clearly to the experimental crossover lengths. Our DFT results for these species are therefore discussed in more detail in the light of possible limitations of the BLYP35+PCM protocol, with the aim of identifying situations where caution needs to be exercised in applying it for the prediction of transport regimes.

On SAMs of $\mathrm{ONI}_{n}$ wires up to $10 \mathrm{~nm}$ long and consisting of alternating fluorene and naphthalene units (see Figure 5), the tunneling-to-hopping crossover was observed at a molecular length of around $4 \mathrm{~nm}$ in the experiments $\left(\mathrm{ONI}_{3}\right.$ to $\left.\mathrm{ONI}_{4}\right) \cdot 18119$ Increasing charge localization is indicated between $\mathrm{ONI}_{3}$ and $\mathrm{ONI}_{4}$ by a strongly decreasing $r_{\text {deloc }}$ from 0.42 to 0.35 , matching the experimental crossover (see Figure 6). In contrast, values of $r_{\text {deloc }}$ ranging from 0.39 to 0.42 for the first two members of the series indicate charge delocalization to a similar extent and therefore the same transport regime. The majority of the spin density is located on one fluorene subunit in any case for $n \geq 3$, which was also found previously in computational studies by the group of Frisbie.19 Although the change in $r_{\text {deloc }}$ is in line with the experimental crossover, an overall rather localized description of the $\mathrm{ONI}_{n}$ wires is provided by our calculations, as indicated by $r_{\text {deloc }}$ never exceeding a value of 0.5 and as also illustrated by the spin densities in Figure 5.

One probable cause for the $\mathrm{ONI}_{n}$ wires showing a rather high degree of charge localization may arise from the isolated-molecule description by our DFT calculations, which cannot capture the spatial confinement resulting from the monolayer present in the experiment. The conformation of the freely moving single wires considered in our calculations might differ from 

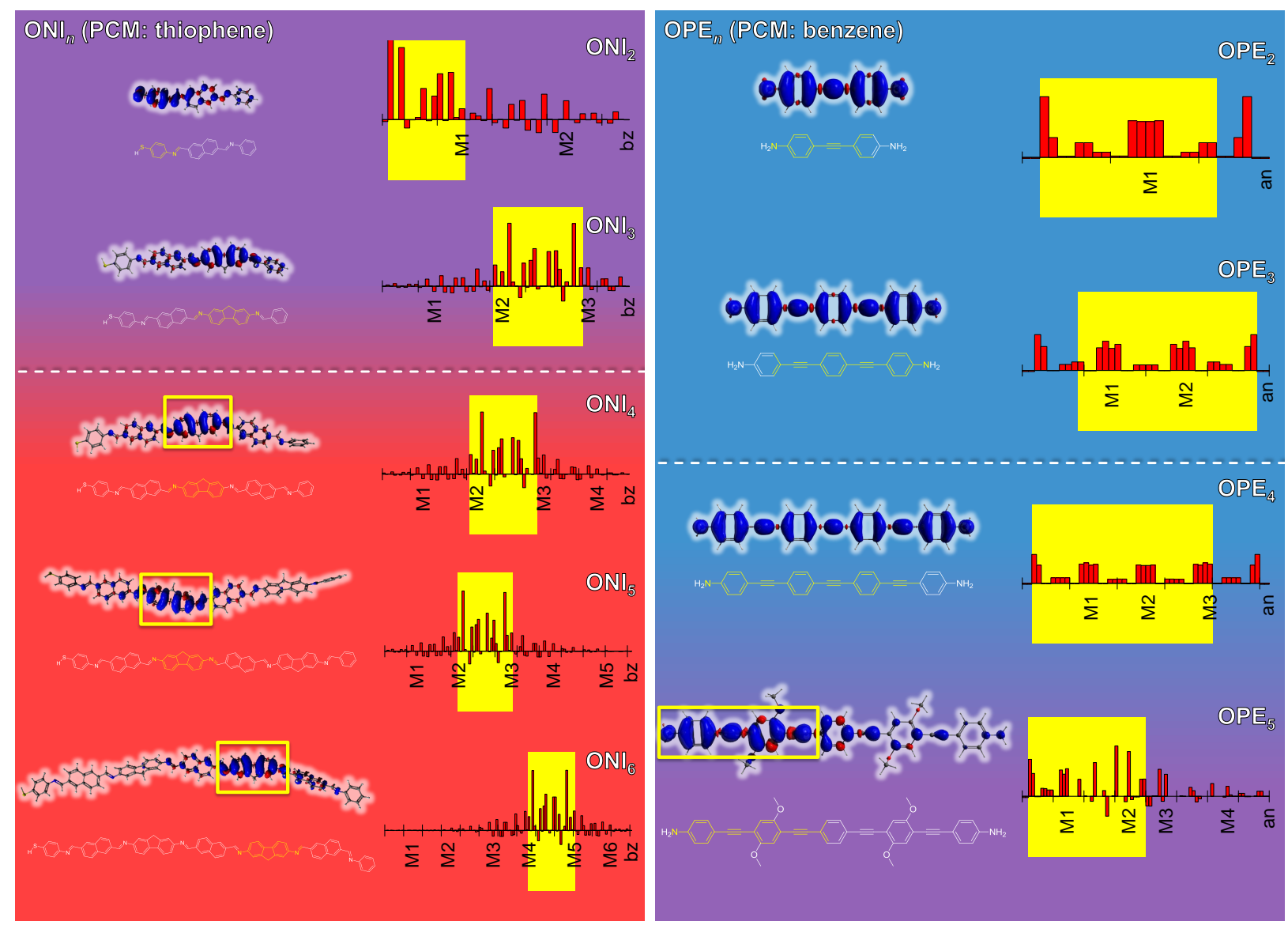

Figure 5: $\mathrm{ONI}_{n}$ (left) and $\mathrm{OPE}_{n}$ (right) radical cationic wires optimized with the BLYP35 functional and Ahlrich's def2-TZVP basis set in thiophene and benzene (PCM). Spin densities and chemical structures of the molecular wires are shown with the local spin density per atom along the molecular backbone (total amount of local spin density $=1$ ). Subregions hosting the majority of the spin density (80-85\%) are highlighted in yellow. The degree of charge delocalization is coded by the background colour (charge delocalization: blue; increasing localization: red). The experimental crossover is indicated by the white dotted line.

the relevant conformations in the experiment in such a way that the degree of charge localization is affected. For example, the electronic properties of conducting polymers strongly depend on their conformation, since torsion angles between adjacent rings determine the magnitude of the overlap between participating molecular orbitals, as pointed out by André and Brédas. ${ }^{[75}$ However, unless torsion angles between adjacent ring units do not exceed a value of 40 degrees, the electronic properties are not expected to be substantially different in comparison to the coplanar situation. ${ }^{75}$ 

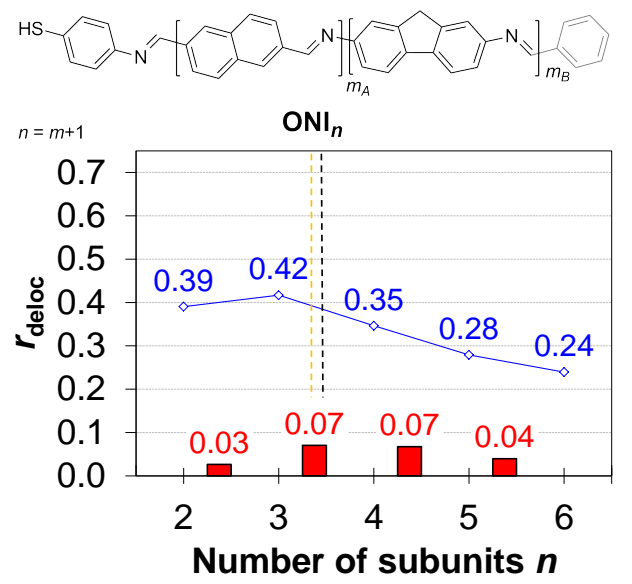

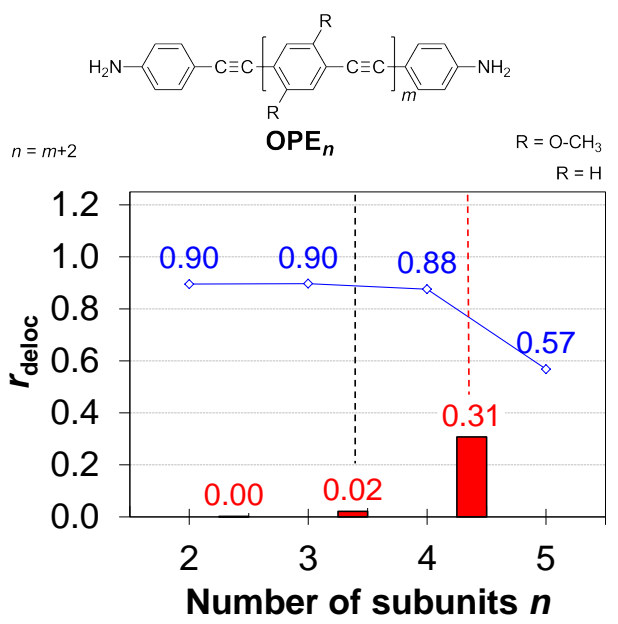

Figure 6: Chemical structures and calculated delocalization measure $r_{\text {deloc }}$ as defined by Equation (1) for ONI (left) and OPE (right) wires as a function of the number of subunits $n$ (blue line) and the corresponding change in $r_{\text {deloc }}$ from subunit $n$ to $n+1$ (red bars). Black dotted line: Experimental crossover from tunneling to hopping. Coloured dotted line: Theoretically predicted crossover based on the calculated delocalization measure (orange: fairly well, red: poor; percentage of spin density: 80-85\%).

As can be seen from Figure 5, the molecular structures of the optimized $\mathrm{ONI}_{n}$ molecules are considerably twisted. Still, torsional angles around the $\mathrm{C}-\mathrm{N}$ bond between adjacent ring units of more than 40 degrees are exclusively present in the longer wires from $\mathrm{ONI}_{3}$ to $\mathrm{ONI}_{6}$, whereas for the shorter $\mathrm{ONI}_{2}$ species they do not exceed 35 degrees (see Supporting Information, Section S2, Table S1).

The twisting of the single-molecule structures in our calculations is likely not occurring to the same degree in the experiments, as their flexibility is limited by the presence of adjacent wires in SAMs, therefore possibly leading to a higher degree of planarization. Moreover, $\pi$ interactions between the relatively large fluorene and napthalene building blocks of adjacent wires may lead to an overall more flattened structure in the latter scenario, resulting in more efficient charge and spin delocalization.

In order to reveal steric effects in a densely packed environment on the molecular structures, the structural optimization or MD simulation of dimers or trimers comprising a small number of wires may provide valuable insight into the mutual impact of molecular wires on 
their structure and therefore their localization properties. Still, despite a rather localized description possibly due to the lack of more detailed environmental modelling, the experimental crossover is correctly described for the $\mathrm{ONI}_{n}$ wires by our DFT approach.

The charge transport characteristics of amine-terminated $\mathrm{OPE}_{n}$ wires up to $5 \mathrm{~nm}$ long were investigated at the single-molecule level using the scanning tunneling microscopy breakjunction (STM-BJ) technique (see Figure 5), 17 where the crossover from tunneling to hopping was observed at a molecular length of around $3 \mathrm{~nm}\left(\mathrm{OPE}_{3}\right.$ to $\left.\mathrm{OPE}_{4}\right)$, see Table 1. For the longer wires, dimethoxyparaphenylene (DMP) units were incorporated into the molecular backbone to increase the solubility without affecting the conductance properties.

For the first three species, $\mathrm{OPE}_{2}$ to $\mathrm{OPE}_{4}$, a relatively high degree of charge delocalization is indicated on the basis of the calculated $r_{\text {deloc }}$ as it ranges from 0.90 to 0.88 . A distinct increase of charge localization is observed with growing molecular length when going from $\mathrm{OPE}_{4}$ to $\mathrm{OPE}_{5}$, as $r_{\text {deloc }}$ decreases from 0.96 to 0.56 .

The increase of charge localization setting in for $\mathrm{OPE}_{5}$ in our calculations is not fully in line with the experiments, where the crossover is observed already one monomer unit earlier for $\mathrm{OPE}_{4}$. Consequently, a slightly overdelocalized description of the $\mathrm{OPE}_{n}$ wires is provided by our DFT calculations when compared to the experiment, which is also indicated by $r_{\text {deloc }}$ of the longest member $\mathrm{OPE}_{5}$ not falling below 0.5. For the latter species, the hopping site comprises roughly three ring units, hosting the majority of the spin density.

The rather delocalized description of the $\mathrm{OPE}_{n}$ wires compared to the experiments might be attributed to discrepancies between the bonding situation of the molecular wire to the electrode surface modeled in our calculations and in the experiments. For the first three members of the series, a shortening of the lengths of the $\mathrm{C}-\mathrm{N}$ bond, connecting the molecular backbone to the amine linker unit, is observed from $1.37 \AA$ to $1.34-1.35 \AA$ on both termini when compared to the molecular structure optimized in the neutral state (see Section S2, Table S2 in Supporting Information). 
In contrast, for the longer $\mathrm{OPE}_{5}$ species, shortening of the $\mathrm{C}-\mathrm{N}$ bond length to $1.34 \AA$ is observed only on one side of the molecule where the charge is predominantly localized. The bond length shortening in our calculations might be caused by $\pi$-backbonding of the free electron pair of the nitrogen atom to the adjacent carbon atom, leading to stronger planarization and therefore facilitating charge delocalization. However, a different situation may prevail in the actual experiments: $\frac{76}{76}$ One can assume that the free electron pair is less strongly donated to the molecular backbone than suggested from the calculations, but is rather binding to the gold electrode surface by the formation of a donor-acceptor bond.77 Still, as pointed out by Venkataraman and coworkers, this bond is weakened due to partial delocalization of the lone pair into the molecular $\pi$-system when the amine group is connected to an aromatic system, such as in case of $\mathrm{OPE}_{n} \cdot{ }^{[77} \mathrm{In}$ our calculations, however, not only partial but full delocalization into the molecule can be assumed due to the lack of the electrode contact.

In case of amines, the molecule-electrode bond is relatively weak when compared to previously discussed molecular wires with different anchoring groups, such as thiols and pyridines, therefore leading to weaker coupling to the gold electrode. As pointed out by van der Zant and coworkers, $\frac{78}{78}$ the formation of so-called image charges is particularly apparent in these weakly coupled molecules, where charges residing on the molecular backbone are screened by the electrodes, consequently leading to long-range polarization effects. ${ }^{79}$ Accordingly, in a theoretical study of Thygesen and Mavrikakis it was found that the dipole moment at the interface of junctions with a diamine anchoring group is larger than for a dithiol group. ${ }^{80}$ As these interface effects, caused by different anchoring groups in the experiments, are not covered in our calculations, the degree of charge localization present in the experiments might be underestimated in our DFT simulations. Interestingly, as discussed in Section 3.1, our DFT results are in good agreement with the experiments in case of the structurally similar $\mathrm{OAE}_{n}$ wires, where the anchoring units are represented by pyridyl linkers instead of amines. In both cases, the crossover is indicated around the same length of $3 \mathrm{~nm}$ by our DFT cal- 
culations, irrespective of the anchoring unit, suggesting that effects on charge localization resulting from the anchoring groups are neglected in our single-molecule calculations.

\section{Conclusions}

In this study, we used a DFT protocol suggested by Renz and Kaupp based on the BLYP35 hybrid functional with a continuum solvent model to evaluate charge localization properties of molecular wires. Our aim was to predict the length-dependent crossover from tunneling to hopping obtained from molecular conductance experiments. $\frac{15[17-19}{19}$ In Table 1, the results of our DFT calculations regarding the crossover from tunneling to hopping are compared to the experiments for the five different series of molecular wires investigated in this study. The theoretical crossover was derived from the change in the delocalization measure $r_{\text {deloc }}$ of consecutive wires with growing bridge length, as defined in Section 2. As a primary criterion, we used the largest relative change, i.e. a sudden drop in $r_{\text {deloc }}$ for consecutive molecular wires as an indicator for increasing charge localization and therefore the crossover from tunneling to hopping. As a secondary criterion, a value of $r_{\text {deloc }}=0.5$ was used as guideline for identifying the crossover length, as the crossover likely occurs in this transition zone from delocalization to localization. Depending on the experimental conditions, the optimization of the molecular structures was performed with linker units either attached to one terminus (SAMs) or on both termini (single-molecule experiments).

Our computational results suggest that the BLYP35+PCM protocol works well not only in describing the general trend of increasing charge localization with growing bridge length, but also correctly predicts the crossover length in most cases. For all wires except for $\mathrm{OPE}_{n}$, we found a good agreement between theory and experiment regarding the transition length when the protocol is used for calculations on single molecular wires ${ }^{4}$.

\footnotetext{
${ }^{4}$ See Section S4.1 in Supporting Information for results on isolated wires without environmental modelling, resulting in an overdelocalized description compared to results from calculations with PCM.
} 
Table 1: Comparison of tunneling-hopping crossover from molecular conductance experiments at room temperature and from DFT calculations with the BLYP35 hybrid functional and Ahlrich's def2-TZVP basis set for various systems. The theoretical crossover is derived from the major change in $r_{\text {deloc }}$ calculated according to Equation (1) (Section 2) and indicating increasing charge localization (percentage of spin density: 80-85\%). Molecular structures are optimized with a PCM as specified.

\begin{tabular}{l|llll|ll}
\hline & \multicolumn{4}{|c}{ Experiment } & \multicolumn{2}{c}{ Theory } \\
\hline & Conditions & Linker & Monomer & Crossover & Crossover & PCM \\
& & & & $n$ & $n$ & \\
\hline $\mathrm{OPTI}_{n}$ & SAMs, vac. & thiol & thiophene/phenylene & $6 \rightarrow 7$ & $6 \rightarrow 7$ & thiophene \\
$\mathrm{OPI}_{n}$ & SAMs, vac. & thiol & phenylene & $5 \rightarrow 6$ & $5 \rightarrow 6$ & benzene \\
$\mathrm{ONI}_{n}$ & SAMs, vac. & thiol & fluorene/naphthalene & $3 \rightarrow 4$ & $3 \rightarrow 4$ & benzene \\
$\mathrm{OAE}_{n}$ & single wires, sol. & pyridyl & phenylene/DMP & $5 \rightarrow 6$ & $5 \rightarrow 6$ & THF \\
$\mathrm{OPE}_{n}$ & single wires, vac. & amine & phenylene/DMP & $3 \rightarrow 4$ & $4 \rightarrow 5$ & benzene
\end{tabular}

When applying the DFT protocol with $\mathrm{PCM}$ to the $\mathrm{ONI}_{n}$ and $\mathrm{OPE}_{n}$ wires, a slightly overlocalized or overdelocalized description is obtained, respectively. Still, for $\mathrm{ONI}_{n}$, the crossover between tunneling and hopping is predicted correctly based on a sudden drop in the delocalization measure, whereas for $\mathrm{OPE}_{n}$ the crossover is delayed by one monomer unit in our calculations compared to the experiment.

The approximate DFT protocol used in this study therefore works well in predicting the experimental crossover in most cases, but needs to be used carefully where effects arising from the electrode-molecule interface are expected to have an influence on charge localization, as some aspects are neglected: First, our calculations lack the description of the gold electrode. In case of amine anchoring groups such as in $\mathrm{OPE}_{n}, \pi$-backbonding of the free electron pair from the nitrogen atom to the molecular backbone rather than binding to the electrode may lead to a more planarized molecular structure in the calculations, suggesting stronger charge delocalization compared to the experiments. Second, amine linkers might induce the formation of larger dipole moments at the molecule-electrode interface when compared to thiols due to weaker coupling to the substrate, as pointed out by Thygesen and Mavrikakis. $\frac{80}{10}$ The formation of image charges might therefore be facilitated, leading to 
long-range polarization effects that are not covered in the calculations.

The neglect of interface effects possibly results in the underestimation of charge localization in the calculations compared to the experiments. For example, for both wires, $\mathrm{OPE}_{n}$ and $\mathrm{OAE}_{n}$, which share a similar molecular backbone but are terminated by different anchoring groups, our DFT protocol predicts the crossover at the same molecular length of around $3 \mathrm{~nm}$ in contrast to the different lenghts found in the experiment. It therefore can be presumed that the effect of the anchoring group on charge localization in the experiments is not fully covered by our DFT protocol.

As mentioned earlier, in our DFT protocol the concept of error compensation may be exploited, based on the idea that the lack of conformational sampling (e.g. by MD simulations), of nonequilibrium effects and of the interface description are likely partially compensated by the approximate DFT protocol. Since the first two factors are dependent on the molecular length and on the experimental setup, i.e. experiments on SAMs or single molecules, it can be assumed that the BLYP35+PCM protocol does not perform consistently well across a broad range of molecular wires of different length and under different experimental conditions.

It has to be noted that the incorporation of a particular correction to a model might not necessarily lead to a more reasonable description. On the example of $\mathrm{OPTI}_{n}$ wires terminated by small gold clusters we showed that the approximate inclusion of both effects, the gold electrode and the environment via PCM, at the same time results in a completely overlocalized description in the calculations and therefore dramatically impairs its performance (see discussion in Section S4.4 in Supporting Information). Therefore, caution needs to be exercised when choosing the parameters that are entering in the calculations, as pointed out by Elstner and Kleinekathöfer. 2728

To conclude, although the transition length is not predicted perfectly in case of $\mathrm{OPE}_{n}$, the DFT protocol still describes not only the increasing trend of charge localization as a 
function of length qualitatively well, but also correctly predicts the crossover from tunneling to hopping with an error of at most one monomer unit, possibly through error compensation. Further improvement might result from employing local hybrid functionals. $\frac{47}{47}$

The approach presented in this study is easily applicable to a variety of conjugated organic molecular wires and has predictive character as it is based on first-principles approaches, at least when applied to molecules studied at room temperature. Since the transport mechanism is not only dependent on the length but also on the temperature, ${ }^{1}$ our approach may be limited to experiments performed in this temperature range.

Our protocol may prove useful not only to gain insight into the charge transport characteristics of a particular molecular system to identify the underlying transport mechanism, but also to reveal the nature and extent of the hopping sites that are involved in the charge transport. The latter aspect is of vital importance, especially in the context of charge transport through biomolecules, e.g. proteins and DNA, which not only strongly depends on the molecular length but also on the architecture of the molecular backbone and thus on the number and sequence of amino acids and base pairs, respectively. 13181

An interesting objective of future studies is therefore to verify the validity of the present DFT protocol in predicting transport mechanisms not only as a function of molecular length, but also of the molecular structure, e.g. the base sequence in DNA. ${ }^{1381}$ Beyond that, it appears worthwile to evaluate semi-empirical methods as a more efficient and therefore promising alternative to the present DFT protocol, as they were previously applied to the investigation of charge transfer in organic mixed-valence systems. ${ }^{82}$ This could be valuable for screening of larger data sets, and would also help to identify efficient electronic structure protocols for use in comprehensive multiscale approaches. $\frac{2728}{27}$ For a more detailed analysis of potential error compensation in our protocol, it may be helpful to explicity compare coherent and hopping charge transport rates and to identify the length-dependent crossover point for a given electronic structure description, as suggested in Ref. $\frac{86}{}$ It would also be 
worthwhile checking whether machine learning methods, as recently successfully applied for predicting transport mechanisms in DNA, ${ }^{87}$ can be transferred to the types of wires under study here. Altogether, such approaches may contribute to a more predictive theoretical framework for molecular and nanoscale electronics.

\section{Acknowledgement}

We thank the high-performance computing center (HPC) at the University of Hamburg for computational resources. We are also thankful for financing by the Deutsche Forschungsgemeinschaft (DFG) through project HE 5675/4-1 and for financial support by the Pro Exzellenzia 4.0 initiative at Hamburg.

\section{A Computational Methodology}

Neutral structures of the molecular wires were preoptimized by performing KS-DFT calculations using the resolution-of-identity (RI) approach, implemented in the TURBOmOLE 7.0 package. ${ }^{88}$ In the molecular structure optimizations, the BP86 exchange-correlation functional, ${ }^{8990}$ Ahlrich's def2-TZVP basis set ${ }^{191}$ of triple-zeta quality with polarization functions on all atoms, and the D3 dispersion correction introduced by Grimme ${ }^{\sqrt{92}}$ were employed. The convergence criterion in the self-consistent field (SCF) algorithm was set to $10^{-7}$ hartree for the change of the energy in all calculations and to $10^{-4}$ a.u. for the gradient in molecular structure optimizations. Subsequent molecular structure optimizations on the neutral preoptimized structures were performed for the molecular wires in their radical cationic state by employing the GAUSSIAN 09 program package ${ }^{93}$ with Ahlrich's def2-TZVP basis set and the hybrid BLYP35 functional with 35\% exact exchange from the Kaupp group, which was constructed analogously to the B1LYP model. ${ }^{94}$ Molecular structure optimizations were 
performed for both the isolated molecules and molecules in solution, since the importance of environmental effects on charge localization properties was pointed out by Kaupp and coworkers and was shown in our previous study on organic mixed-valence systems. ${ }^{46 / 48}$ For the inclusion of solvent effects, the polarizable continuum model with the integral equation formalism model (IEFPCM $)^{95[96}$ was employed as implemented in the GAUSSIAN 09 program package by using the SCRF keyword with the available dielectric constants for thiophene $(\epsilon=2.7270)$, benzene $(\epsilon=2.2706)$ and tetrahydrofuran $(\epsilon=7.4257)$. Natural population analyses ${ }^{97}$ were performed with the GAUSSIAN 09 program package to gain information about the distribution of local spin densities and charges, which were summed over specific subregions of the molecule. For the local spin density, an absolute value of 1 refers to one unpaired electron (i.e., a more precise yet more cumbersome expression would be "local unpaired electron density"). Molecular structures and spin densities were visualized with the Avogadro editor, $\frac{98}{9}$ applying an isosurface value of 0.001 for plotting the spin density distributions.

\section{References}

(1) Cuevas, J. C.; Scheer, E. In Molecular Electronics: An Introduction to Theory and Experiment, 2nd ed.; Reed, M., Ed.; Nanoscience and Nanotechnology; World Scientific, 2017; Vol. 1.

(2) Garner, M. H.; Li, H.; Chen, Y.; Su, T.; Shangguan, Z.; Paley, D.; Liu, T.; Ng, F.; Li, H.; Xiao, S.; Nuckolls, C.; Venkataraman, L.; Solomon, G. Comprehensive suppression of single-molecule conductance using destructive $\sigma$-interference. Nature 2018, 558, 415419.

(3) Jones, L. O.; Mosquera, M. A.; Fu, B.; Schatz, G. C.; Marks, T. J.; Ratner, M. A. Quantum Interference and Substantial Property Tuning in Conjugated Z-ortho-Regio- 
Resistive Organic (ZORRO) Junctions. Nano Letters 2019, 19, 8956-8963, PMID: 31682761.

(4) Garner, M. H.; Koerstz, M.; Jensen, J. H.; Solomon, G. C. The Bicyclo[2.2.2]octane Motif: A Class of Saturated Group 14 Quantum Interference Based Single-Molecule Insulators. J. Phys. Chem. Lett. 2018, 9, 6941-6947.

(5) Mtangi, W.; Kiran, V.; Fontanesi, C.; Naaman, R. Role of the Electron Spin Polarization in Water Splitting. J. Phys. Chem. Lett. 2015, 6, 4916-4922.

(6) Naaman, R.; Paltiel, Y.; Waldeck, D. H. Chiral molecules and the electron spin. Nature Reviews 2019, 3, 250260.

(7) Abendroth, J. M.; Cheung, K. M.; Stemer, D. M.; Hadri, M. S. E.; Zhao, C.; Fullerton, E. E.; Weiss, P. S. Spin-Dependent Ionization of Chiral Molecular Films. J. Am. Chem. Soc. 2019, 141, 3863-3874.

(8) Aragones, A. C.; Medina, E.; Ferrer-Huerta, M.; Gimeno, N.; Teixido, M.; Palma, J. L.; Tao, N.; Ugalde, J. M.; Giralt, E.; Diez-Perez, I.; Mujica, V. Measuring the SpinPolarization Power of a Single Chiral Molecule. small 2017, 13, 1602519.

(9) Kettner, M.; Maslyuk, V. V.; Nrenberg, D.; Seibel, J.; Gutierrez, R.; Cuniberti, G.; Ernst, K.-H.; Zacharias, H. Chirality-Dependent Electron Spin Filtering by Molecular Monolayers of Helicenes. J. Phys. Chem. Lett. 2018, 9, 2025-2030.

(10) Maslyuk, V. V.; Gutierrez, R.; Dianat, A.; Mujica, V.; Cuniberti, G. Enhanced Magnetoresistance in Chiral Molecular Junctions. J. Phys. Chem. Lett. 2018, 9, 5453-5459.

(11) Zöllner, M. S.; Varela, S.; Medina, E.; Mujica, V.; Herrmann, C. Insight into the Origin of Chiral-Induced Spin Selectivity from a Symmetry Analysis of Electronic Transmission. J. Chem. Theory Comput. 16, 2914-2929.

(12) Heath, J. R.; Ratner, M. A. Molecular electronics. Physics Today 2003, 56, 43-49. 
(13) Giese, B.; Amaudrut, J.; Köhler, A.-K.; Spormann, M.; Wessely, S. Direct observation of hole transfer through DNA by hopping between adenine bases and by tunnelling. Nature 2001, 412, 318-320.

(14) Kawanishi, S.; Hiraku, Y.; Oikawa, S. Mechanism of guanine-specific DNA damage by oxidative stress and its role in carcinogenesis and aging. Mutat. Res. 2001, 488, 65-76.

(15) Smith, C. E.; Odoh, S. O.; Ghosh, S.; Gagliardi, L.; Cramer, C. J.; Christopher, J. K.; Frisbie, D. C. Length-Dependent Nanotransport and Charge Hopping Bottlenecks in Long Thiophene-Containing $\pi$-Conjugated Molecular Wires. J. Am. Chem. Soc. 2015, 137, 15732-15741.

(16) Zhao, X.; Huang, C.; Gulcur, M.; Batsanov, A. S.; Baghernejad, M.; Hong, W.; Bryce, M. R.; Wandlowski, T. Oligo(aryleneethynylene)s with Terminal Pyridyl Groups: Synthesis and Length Dependence of the Tunneling-to-Hopping Transition of SingleMolecule Conductances. Chem. Mater. 2013, 25, 4340-4347.

(17) Lu, Q.; Liu, K.; Zhang, H.; Du, Z.; Wang, X.; Wan, F. From Tunneling to Hopping: A Comprehensive Investigation of Charge Transport Mechanism in Molecular Junctions Based on Oligo(p-phenylene ethynylene)s. ACS Nano 2009, 3, 3861-3868.

(18) Choi, S. H.; Kim, B.; Frisbie, C. D. Electrical Resistance of Long Conjugated Molecular Wires. Science 2008, 320, 1482-1486.

(19) Choi, S. H.; Risko, C.; Delgado, M. C. R.; Kim, B.; Bredas, J.-L.; Frisbie, C. D. Transition from Tunneling to Hopping Transport in Long, Conjugated Oligo-imine Wires Connected to Metals. J. Am. Chem. Soc. 2010, 132, 4358-4368.

(20) Solomon, G. C.; Herrmann, C.; Ratner, M. A. In Molecular Electronic Junction Transport: Some Pathways and Some Ideas. In: Unimolecular and Supramolecular Electronics II. Topics in Current Chemistry; Metzger, R., Ed.; Springer, Berlin, Heidelberg, 2011; Vol. 313. 
(21) Kamenetska, M.; Widawsky, J. R.; Dell'Angela, M.; Frei, M.; Venkataraman, L. Temperature dependent tunneling conductance of single molecule junctions. J. Chem. Phys. 2017, 146, 092311.

(22) Nitzan, A. Electron Transmission through molecules and molecular interfaces. Annu. Rev. Phys. Chem. 2001, 52, 681-750.

(23) Xiang, L.; Palma, J. L.; Bruot, C.; Mujica, V.; Ratner, M. A.; Tao, N. Intermediate tunnelling-hopping regime in DNA charge transport. Nat. Chem. 2015, 7, 221-226.

(24) Xiang, L.; Palma, J. L.; Bruot, C.; Mujica, V.; Ratner, M. A.; Tao, N. Corrigendum: Intermediate tunnelling-hopping regime in DNA charge transport. Nature Chem. 2017, 9, 295-295.

(25) Kim, H.; Kilgour, M.; Segal, D. Intermediate CoherentIncoherent Charge Transport: DNA as a Case Study. J. Phys. Chem. C 2016, 120, 23951-23962.

(26) Renaud, N.; Berlin, Y. A.; Lewis, F. D.; Ratner, M. A. Between Superexchange and Hopping: An Intermediate Charge-Transfer Mechanism in Poly(A)-Poly(T) DNA Hairpins. J. Am. Chem. Soc. 2013, 135, 3953-3963.

(27) Wolter, M.; Elstner, M.; Kleinekathöfer, U.; Kubar̆, T. Microsecond Simulation of Electron Transfer in DNA: Bottom-Up Parametrization of an Efficient Electron Transfer Model Based on Atomistic Details. J. Phys. Chem. B 2017, 121, 529-549.

(28) Kubar̆, T.; Elstner, M.; Popescu, B.; Kleinekathöfer, U. Polaron Effects on Charge Transport through Molecular Wires: A Multiscale Approach. J. Chem. Theory Comput. 2017, 286296.

(29) Oberhofer, H.; Reuter, K.; Blumberger, J. Charge Transport in Molecular Materials: An Assessment of Computational Methods. Chem. Rev. 2017, 117, 10319-10357. 
(30) Xie, W.; Holub, D.; Kubař, T.; Elstner, M. Performance of Mixed Quantum-Classical Approaches on Modeling the Crossover from Hopping to Bandlike Charge Transport in Organic Semiconductors. J. Chem. Theory Comput. 2020, 16, 2071-2084.

(31) Troisi, A. Charge transport in high mobility molecular semiconductors: classical models and new theories. Chem. Soc. Rev. 2011, 40, 2347.

(32) Troisi, A. The speed limit for sequential charge hopping in molecular materials. Org. Elec. 2011, 12, 1988-1991.

(33) Fratini, S.; Mayou, D.; Ciuchi, S. The Transient Localization Scenario for Charge Transport in Crystalline Organic Materials. Adv. Funct. Mater. 2016, 26, 2292-2315.

(34) Leitherer, S.; Jäger, C. M.; Krause, A.; Halik, M.; Clark, T.; Thoss, M. Simulation of charge transport in organic semiconductors: A time-dependent multiscale method based on nonequilibrium Greens functions. Phys. Rev. Mater. 2017, 1, 064601.

(35) Nematiaram, T.; Troisi, A. Modeling charge transport in high-mobility molecular semiconductors: Balancing electronic structure and quantum dynamics methods with the help of experiments. J. Chem. Phys. 2020, 152, 190902.

(36) Sowa, J. K.; Mol, J. A.; Briggs, G. A. D.; Gauger, E. M. Beyond Marcus theory and the Landauer-Büttiker approach in molecular junctions: A unified framework. J. Chem. Phys. 2018, 149, 154112.

(37) Carey, R.; Chen, L.; Gu, B.; Franco, I. When can time-dependent currents be reproduced by the Landauer steady-state approximation? J. Chem. Phys. 2017, 146, 174101.

(38) Gu, B.; Franco, I. Generalized Theory for the Timescale of Molecular Electronic Decoherence in the Condensed Phase. J. Phys. Chem. Lett. 2018, 9, 773-778. 
(39) Gu, B.; Franco, I. Correction to "Generalized Theory for the Timescale of Molecular Electronic Decoherence in the Condensed Phase". J. Phys. Chem. Lett. 2020, 11, 20442045.

(40) Thoss, M.; Evers, F. Perspective: Theory of quantum transport in molecular junctions. J. Chem. Phys. 2018, $148,030901$.

(41) Chen, S.; Zhang, Y.; Koo, S.; Tian, H.; Yam, C.; Chen, G.; Ratner, M. A. Interference and Molecular Transport — A Dynamical View: Time-Dependent Analysis of Disubstituted Benzenes. J. Phys. Chem. Lett. 2014, 5, 2748.

(42) Kim, H.; Segal, D. Controlling charge transport mechanisms in molecular junctions: Distilling thermally induced hopping from coherent-resonant conduction. J. Chem. Phys. 2017, 146, 164702.

(43) Kilgour, M.; Segal, D. Charge transport in molecular junctions: From tunneling to hopping with the probe technique. J. Chem. Phys. 2015, 143, 024111.

(44) Renz, M.; Kaupp, M. Predicting the Localized/Delocalized Character of Mixed-Valence Diquinone Radical Anions. Toward the Right Answer for the Right Reason. J. Phys. Chem. A 2012, 116, 10629-10637.

(45) Renz, M.; Kess, M.; Diedenhofen, M.; Klamt, A.; Kaupp, M. Reliable Quantum Chemical Prediction of the Localized/Delocalized Character of Organic Mixed-Valence Radical Anions. From Continuum Solvent Models to Direct-COSMO-RS. J. Chem. Theory Comput. 2012, 8, 4189-4203.

(46) Parthey, M.; Kaupp, M. Quantum-chemical insights into mixed-valence systems: within and beyond the Robin-Day scheme. Chem. Soc. Rev. 2014, 43, 5067-5088.

(47) Maier, T. M.; Arbuznikov, A. V.; Kaupp, M. Local hybrid functionals: Theory, im- 
plementation, and performance of an emerging new tool in quantum chemistry and beyond. WIREs Comput. Mol. Sci. 2018, in press, DOI 10.1002/wcms.1378.

(48) Kröncke, S.; Herrmann, C. Designing Long-Range Charge Delocalization from FirstPrinciples. J. Chem. Theory Comput. 2019, 15, 165-177.

(49) Kubař, T.; Elstner, M. Efficient algorithms for the simulation of non-adiabatic electron transfer in complex molecular systems: application to DNA. Physical Chemistry Chemical Physics 2013, 15, 5794-5813.

(50) Kubař, T.; Elstner, M. A hybrid approach to simulation of electron transfer in complex molecular systems. Journal of The Royal Society Interface 2013, 10, 20130415.

(51) Dong, B.; Ding, G. H.; Lei, X. L. Time-dependent quantum transport through an interacting quantum dot beyond sequential tunneling: second-order quantum rate equations. Journal of Physics: Condensed Matter 2015, 27, 205303.

(52) Woiczikowski, P. B.; Kubař, T.; Gutierrez, R.; Cuniberti, G.; Elstner, M. Structural stability versus conformational sampling in biomolecular systems: Why is the charge transfer efficiency in G4-DNA better than in double-stranded DNA? The Journal of Chemical Physics 2010, 133, 035103.

(53) Giannini, S.; Carof, A.; Blumberger, J. Crossover from Hopping to Band-Like Charge Transport in an Organic Semiconductor Model: Atomistic Nonadiabatic Molecular Dynamics Simulation. J. Phys. Chem. Lett. 2018, 9, 3116-3123.

(54) Giannini, S.; Carof, A.; Ellis, M.; Yang, H.; Ziogos, O. G.; Ghosh, S.; Blumberger, J. Quantum localization and delocalization of charge carriers in organic semiconducting crystals. Nat. Commun. 2019, 10,1-12.

(55) Li, G.; Movaghar, B.; Nitzan, A.; Ratner, M. A. Polaron formation: Ehrenfest dynamics vs. exact results. J. Chem. Phys. 2013, 138, 044112. 
(56) Li, G.; Govind, N.; Ratner, M. A.; Cramer, C. J.; Gagliardi, L. Influence of Coherent Tunneling and Incoherent Hopping on the Charge Transfer Mechanism in Linear DonorBridge-Acceptor Systems. J. Phys. Chem. 2015, 6, 4889-4897.

(57) Franco, C.; Burrezo, P. M.; Lloveras, V.; Caballero, R.; Alcn, I.; Bromley, S. T.; MasTorrent, M.; Langa, F.; Lpez Navarrete, J. T.; Rovira, C.; Casado, J.; Veciana, J. Operative Mechanism of Hole-Assisted Negative Charge Motion in Ground States of Radical-Anion Molecular Wires. J. Am. Chem. Soc. 2017, 139, 686-692.

(58) Fung, E.-D.; Gelbwaser, D.; Taylor, J.; Low, J.; Xia, J.; Davydenko, I.; Campos, L. M.; Marder, S.; Peskin, U.; Venkataraman, L. Breaking Down Resonance: Nonlinear Transport and the Breakdown of Coherent Tunneling Models in Single Molecule Junctions. Nano Lett. 2019, 19, 2555-2561.

(59) Hankache, J.; Wenger, O. S. Organic Mixed Valence. Chem. Rev. 2011, 111, 5138-5178.

(60) Heckmann, A.; Lambert, C. Organic Mixed-Valence Compounds: A Playground for Electrons and Holes. Angew. Chem. Int. Ed. 2012, 51, 326-392.

(61) Neugebauer, J.; Hess, B. A. Fundamental vibrational frequencies of small polyatomic molecules from density-functional calculations and vibrational perturbation theory. The Journal of Chemical Physics 2003, 118, 7215-7225.

(62) Obersteiner, V.; Egger, D. A.; Zojer, E. Impact of Anchoring Groups on Ballistic Transport: Single Molecule vs Monolayer Junctions. J. Phys. Chem. C 2015, 119, 2119821208.

(63) Hofmann, O. T.; Egger, D. A.; Zojer, E. Work-Function Modification beyond Pinning: When Do Molecular Dipoles Count? Nano Lett. 2010, 10, 4369-4374.

(64) Heimel, G.; Romaner, L.; Zojer, E.; Bredas, J.-L. The Interface Energetics of SelfAssembled Monolayers on Metals. Acc. Chem. Res. 2008, 41, 721-729. 
(65) Herrmann, C.; Reiher, M.; Hess, B. A. Comparative analysis of local spin definitions. J. Chem. Phys. 2005, 122, 034102.

(66) Reed, A. E.; Weinstock, R. B.; Weinhold, F. Natural population analysis. J. Chem. Phys. 1984, 83, 735-746.

(67) Lazić, P.; Alaei, M.; Atodiresei, N.; Caciuc, V.; Brako, R.; Blügel, S. Density functional theory with nonlocal correlation: A key to the solution of the CO adsorption puzzle. Phys. Rev. B 2010, 81, 045401.

(68) Janthon, P.; Vines, F.; Sirijaraensre, J.; Limtrakul, J.; Illas, F. Adding Pieces to the CO/Pt(111) Puzzle: The Role of Dispersion. J. Phys. Chem. C 2017, 121, 3970-3977.

(69) Patra, A.; Peng, H.; Sun, J.; Perdew, J. P. Rethinking CO adsorption on transitionmetal surfaces: Effect of density-driven self-interaction errors. Phys. Rev. B 2019, 100, 035442 .

(70) Bahlke, M. P.; Wahl, P.; Diekhöner, L.; Herrmann, C. Co(CO $)_{n} / \mathrm{Cu}(001)$ : Towards understanding chemical control of the Kondo effect. J. Appl. Phys. 2019, 125, 142910.

(71) Liu, Z.-F.; Egger, D. A.; Refaely-Abramson, S.; Kronik, L.; Neaton, J. B. Energy level alignment at molecule-metal interfaces from an optimally tuned range- separated hybrid functional. J. Chem. Phys. 2017, 146, 092326.

(72) Liu, Z.-F.; da Jornada, F. H.; Louie, S. G.; Neato, J. B. Accelerating GW-Based Energy Level Alignment Calculations for MoleculeMetal Interfaces Using a Substrate Screening Approach. J. Chem. Theory Comput. 2019, 15, 4218-4227.

(73) Thygesen, K. S.; Rubio, A. Renormalization of Molecular Quasiparticle Levels at MetalMolecule Interfaces: Trends across Binding Regimes. Phys. Rev. Lett. 2009, 102, 046802. 
(74) Neaton, J. B.; Hybertsen, M. S.; Louie, S. G. Renormalization of Molecular Electronic Levels at Metal-Molecule Interfaces. Phys. Rev. Lett. 2006, 97, 216405.

(75) Brédas, J. L.; Street, G. B.; Thémans, B.; André, J. M. Organic polymers based on aromatic rings (polyparaphenylene, polypyrrole, polythiophene): Evolution of the electronic properties as a function of the torsion angle between adjacent rings. J. Chem. Phys. 1985, 1323.

(76) Stuyver, T.; Zeng, T.; Tsuji, Y.; Fias, S.; Geerlings, P.; de Proft, F. Captodative Substitution: A Strategy for Enhancing the Conductivity of Molecular Electronic Devices. J. Phys. Chem. C 2018, 122, 3194-3200.

(77) Frei, M.; Aradhya, S. V.; Koentopp, M.; Hybertsen, M. S.; Venkataraman, L. Mechanics and Chemistry: Single Molecule Bond Rupture Forces Correlate with Molecular Backbone Structure. Nano Lett. 2011, 11, 1518-1523.

(78) Perrin, M. L.; Verzijl, C. J. O.; Martin, C. A.; Shaikh, A. J.; Eelkema, R.; van Esch, J. H.; van Ruitenbeek, J. M.; Thijssen, J. M.; van der Zant, H. S. J.; ; Dulic, D. Large tunable image-charge effects in single-molecule junctions. Nat. Nanotechnol. 2013, 8 .

(79) Strange, M.; Thygesen, K. Image-charge-induced localization of molecular orbitals at metal-molecule interfaces: Self-consistent GW calculations. Phys. Rev. B 2012, 86, 195121.

(80) Peng, G.; Strange, M.; Thygesen, K. S.; Mavrikakis, M. Conductance of Conjugated Molecular Wires: Length Dependence, Anchoring Groups, and Band Alignment. J. Phys. Chem. C 2009, 113, 20967-20973.

(81) Tao, N. J. Electron transport in molecular junctions. Nat. Nanotechnol. 2006, 1, 173181. 
(82) Lacroix, J. C.; Chane-Ching, K. I.; Maquére, F.; Maurel, F. Intrachain Electron Transfer in Conducting Oligomers and Polymers: The Mixed Valence Approach. J. Am. Chem. Soc. 2006, 128, 7264-7276.

(83) van Haare, J. A. E. H.; Havinga, E. E.; van Dongen, J. L. J.; Janssen, R. A. J.; Cornil, J.; Brédas, J.-L. Redox States of Long Oligothiophenes : Two Polarons on a Single Chain. Chem. Eur. J. 1998, 4, 1509.

(84) Barlow, S.; Risko, C.; Chung, S.-J.; Tucker, N. M.; Coropceanu, V.; Jones, S. C.; Levi, Z.; Brédas, J.-L.; Marder, S. R. Intervalence Transitions in the Mixed-Valence Monocations of Bis(triarylamines) Linked with Vinylene and Phenylene-Vinylene Bridges. J. Am. Chem. Soc. 2005, 127, 16900-16911.

(85) Lambert, C.; Nöll, G. The Class II/III Transition in Triarylamine Redox Systems. J. Am. Chem. Soc. 1999, 121, 8434.

(86) Kastlunger, G.; Stadler, R. Density functional theory based direct comparison of coherent tunneling and electron hopping in redox-active single-molecule junctions. Phys. Rev. B 2015, 91, 125410 .

(87) Korol, R.; Segal, D. Machine Learning Prediction of DNA Charge Transport. J. Phys. Chem. B 2019, 123, 2801-2811.

(88) Ahlrichs, R. et al. Turbomole V7.0; software, 2015.

(89) Becke, A. D. Density-functional exchange-energy approximation with correct asymptotic-behavior. Phys. Rev. A 1988, 38, 3098-3100.

(90) Perdew, J. P. Density-functional approximation for the correlation energy of the inhomogeneous electron gas. Phys. Rev. B 1986, 33, 8822.

(91) Weigend, F.; Ahlrichs, R. Balanced basis sets of split valence, triple zeta valence and 
quadruple zeta valence quality for $\mathrm{H}$ to Rn: Design and assessment of accuracy. Phys. Chem. Chem. Phys. 2005, 7, 3297-305.

(92) Grimme, S. Semiempirical GGA-type density functional constructed with a long-range dispersion correction. J. Comput. Chem. 2006, 27, 1787-1799.

(93) Frisch, M. J. et al. Gaussian 09 Revision A.1.; software, 2009.

(94) Adamo, C.; Barone, V. Toward reliable adiabatic connection models free from adjustable parameters. Chem. Phys. Lett. 1997, 274, 242-250.

(95) Miertuš, S.; Scrocco, E.; Tomasi, J. Electrostatic Interaction of a Solute with a Continuum. A Direct Utilization of ab initio Molecular Potentials for the Prevision of Solvent Effects. Chem. Phys. 1981, 55, 117-129.

(96) Cossi, M.; Barone, V.; Cammi, R.; Tomasi, J. Ab initio study of solvated molecules: A new implementation of the polarizable continuum model. Chem. Phys. Lett. 1996, 255, 327-335.

(97) Reed, A. E.; Weinstock, R. B.; Weinhold, F. Natural population analysis. J. Chem. Phys. 1984, 83, 735-746.

(98) Hanwell, M.; Curtis, D.; Lonie, D.; Vandermeersch, T.; Zurek, E.; Hutchison, G. Avogadro: an advanced semantic chemical editor, visualization, and analysis platform. $J$. Cheminf. 2012, 4, 1-17. 